\title{
La nomination des enfants décédés en bas-âge et de leurs parents. D'une analyse du discours située à une linguistique d'intervention.
}

\author{
Giuditta Caliendo ${ }^{1, *}$ et Catherine Ruchon ${ }^{2 \dagger}$ \\ ${ }^{1}$ Université de Lille, STL, UMR 8163 \\ 2Université Sorbonne Paris Nord, PLEIADE, UR 7338
}

\begin{abstract}
Résumé. Cette contribution pose une réflexion sur la nomination des enfants décédés en bas âge et de leurs parents pour lesquels la langue française ne dispose d'aucun terme. Cette question ontologique soulève un problème politique et moral. La lacune lexicale exerce une forme d'oppression par la non reconnaissance de l'enfant décédé et par une perte de statut de parent pour le père et la mère. Un continuum se dessine depuis l'absence d'une désignation dans le lexique jusqu'à la présence de désignations stigmatisantes, dans le discours médico-juridique par exemple. Cet article souhaite ouvrir la voie à une politique de nomination qui associe de manière contributive les personnes concernées à la réflexion discursive et lexicologique. Notre approche s'inscrit dans un double ancrage théorique, celui de l'analyse du discours et celui de l'intersectionnalité. Nous étudierons dans un premier temps les métadiscours des personnes endeuillées en faisant l'inventaire des autodésignations utilisées par les concerné·e·s. Nous montrerons ensuite que certain'e-s d'entre elles'eux tentent de faire entendre leur voix par une action publique et des demandes de réflexion terminologique. Puis nous soulignerons l'activité métalinguistique des énonciatrices teurs dans leur quête d'un désignant adapté à leur situation. L'une des caractéristiques spécifiques de ce travail est la collaboration entre chercheurs -ses et personnes concernées, source de points de vue situés. L'objectif final de cette recherche est la création ou la validation d'une désignation acceptée par les endeuillé $\cdot e \cdot s$.
\end{abstract}

Mots clés : analyse du discours intersectionnelle, désignation, empowerment, folk linguistique, lacune lexicale, point de vue situé.

\begin{abstract}
Designating deceased babies and their parents. From Situated Discourse Analysis to Intervention Linguistics. This paper sets out to investigate the designation of deceased babies and of their parents in the French language, which lacks a specific term to refer to either of them. Such ontological issue triggers a political and moral problem. This lexical gap can translate into forms of oppression (i.e. not acknowledging the deceased baby means
\end{abstract}

\footnotetext{
* Corresponding author : giuditta.caliendo@univ-lille.fr

+ Corresponding author : ruchon@free.fr
} 
depriving their mother and father of their status as parents), or even lead to the emergence of stigmatizing designating terms, for instance in medico-legal discourse. Our paper intends to encourage a new designation policy that is based on the fruitful involvement of all the actors concerned in the relevant discursive and lexicological discussion. Our approach is couched in the double theoretical framework of discourse analysis and intersectionality. The first part of the study is devoted to the metadiscourse of bereaved parents through an inventory of the self-designating terms they use. We will also observe how some of them are attempting to make their voice heard via petitions or by encouraging a public terminological debate. The second stage of our research foregrounds the speakers' metalinguistic activity in their search for an appropriate term to designate their status. The innovative aspect of this work lies in the collaboration between the authors and the actors directly concerned by perinatal death, who are sources of situated viewpoints, with the final aim of creating or validating a designating term that is deemed acceptable.

Keywords: Intersectional discourse analysis, designation, empowerment, folk linguistics, lexical gap, situated viewpoint.

\section{Introduction}

Notre objectif est de réfléchir à la question de la nomination des enfants décédés en bas âge et de leurs parents. Nous partons d'un constat : l'absence de désignation lexicalisée pour l'enfant décédé ou le parent endeuillé (de type [Veuve + Npr]). Celle-ci se traduit en forme d'oppression par une non reconnaissance de l'enfant décédé et simultanément par une perte de statut de parent pour le père et la mère. Un continuum se dessine depuis l'absence d'une désignation dans le lexique jusqu'à la présence de désignations stigmatisantes dans le discours médico-juridique par exemple, comme celles de « déchets », «faux mort-né » ou " produit embryonnaire » en France pour désigner le bébé décédé ou « restes de grossesse » (« remains of pregnancy ») au Royaume-Uni. Cette question soulève un problème politique et moral. Comment élaborer une "politique de la nomination », contribuer, en tant que femmes analystes du discours, à la question soulevée par les endeuillé·e·s dans leurs discours (pouvoir désigner en discours leurs enfants décédés et maintenir leur identité de parent)?

Cette question ontologique concerne chacun et chacune. On ne peut se contenter d'imposer une hétérodésignation forgée par des linguistes non concernés personnellement. Tout au contraire, nous souhaitons nous appuyer sur les savoirs produits par les endeuillé·e·s et les impliquer dans la réflexion discursive et lexicologique.

Comment matérialiser cette réflexion commune ? Nous nous plaçons dans le cadre théorique d'une analyse de discours située et intersectionnelle. Celle-ci se réclame des théories féministes qui refusent la généralisation du point de vue masculin, ce qui a amené à la prise en compte de différents points de vue. La féministe Donna Haraway (1988, 2007) insiste sur cette nécessité de multiplier les points de vue afin de déconstruire les dualismes féminin/masculin, mais aussi naturel/artificiel ou animal/humain. Une philosophe éthologue comme Vinciane Despret $(2009,2019)$ nous invite à prendre le point de vue de l'animal, à « penser comme un rat » ou à « habiter en oiseau ». Il est facile en effet en recherche de généraliser des points de vue particuliers, de parler à la place des femmes, des enfants, des personnes âgées, des personnes en situation de vulnérabilité... 
Les théories féministes nous enseignent comment prendre en considération le caractère « situé » du point de vue en partant d'une situation vécue (Dorlin 2008). De ce fait il est vain de vouloir donner une définition fixe de l'intersectionnalité. Nous travaillerons l'intersectionnalité pour ce qu'elle est, un savoir situé (Bilge 2015, cité par Kocadost 2017, en ligne), en l'appliquant au contexte et au terrain du deuil d'enfant, avec la participation des parents (le plus souvent des mères) ayant perdu un enfant (le plus souvent en bas âge), à partir de notre propre position de femme ayant aussi été concernée par le deuil périnatal. Notre démarche est politique, dans le sens où nous souhaitons sortir les problématiques du deuil (de la nomination en particulier) de la sphère familiale et privée. Les personnes ayant perdu leur enfant sont en effet en partie invisibilisées, sous l'effet d'une doxa d'acceptation, des stéréotypes de l'indicible, et du tabou sur la mort de l'enfant (Heazell 2016 : 388), cela en dépit du taux élevé de mortalité infantile en France (soit 3,8 pour 1000 enfants nés vivants en $2019^{1}$ ).

Pour rendre la théorie fonctionnelle, nous nous intéressons tout particulièrement aux discours métalinguistiques, autrement dit les discours sur la langue produits par des usagers non linguistes de la langue. Ce type de discours appartient à la linguistique dite populaire ou folk. Les observables nécessaires à cette étude ont été prélevés sur un corpus de discours de parents endeuillés publiés sur les forums de discussion consacrés à la perte de l'enfant publiés entre 2004 et 2013, ainsi que dans les bulletins de deux associations (Jonathan pierres Vivantes et Petite Émilie) publiés entre 2006 et 2013, une dizaine d'ouvrages-témoignage de parents endeuillés publiés en France des années 1990 à 2013 et trois pétitions citoyennes créées dans les années 2010 sur la lacune lexicale.

Les discours des parents endeuillés révèlent une réflexion métalinguistique sur l'absence d'un mot pour les désigner et une grande créativité lexicale pour combler cette lacune. Nous présenterons ces différentes désignations en tentant de ne pas imposer notre point de vue de chercheuses sur ces néologismes, le choix final ne pouvant revenir qu'aux concernéee-s. Cet article présente la première étape d'une étude plus large dont la seconde phase consistera à mobiliser les parents endeuillés autour d'une réflexion commune sur le choix ou la création d'une désignation des parents endeuillés (et dans le prolongement, d'une désignation pour l'enfant décédé et pour l'enfant naissant à la suite d'un enfant décédé). Cette méthodologie relève de l'empowerment. Selon William Ninacs, qui a consacré sa thèse à ce sujet, «Le concept d'empowerment correspond à un point de convergence des philosophies axées sur l'idée que les individus et les collectivités ont le droit de participer aux décisions qui les concernent. »(Ninacs 2002 : 50). Le terme apparaît la première fois aux États-Unis au début du $\mathrm{XX}^{\mathrm{e}}$ siècle dans les discours des femmes féministes luttant pour la reconnaissance de leurs droits. La notion a été reprise dans les années 1960 par les spécialistes en psychologie communautaire, en particulier par le québécois Yann Le Bossé (2004), qui a longuement travaillé la notion dans le champ des pratiques sociales, et qui a traduit le terme anglais par la périphrase « développement du pouvoir d'agir des personnes et des collectivités ». Il s'agit selon lui d'un «processus de développement d'un pouvoir individuel et collectif sur l'environnement». Du Québec, la notion migre en France au début des années 2000 et apparaît comme un " modèle-type de démocratie participative » (Bacqué et Biewener 2013). Plus précisément pour ce qui nous concerne, le terme s'applique selon Margot Breton (2000) à des pratiques qui permettent aux exclus de sortir du silence et de participer aux décisions sociopolitiques qui affectent leur vie. La notion d'empowerment est encore très peu convoquée par les linguistes. Elle est pourtant d'un grand soutien dans les études portant sur des terrains et des «corpus sensibles » (Paveau et Perea 2012/2015). Elle apparaît nécessaire dès lors qu'entre en jeu l'acte de nommer des personnes en situation de vulnérabilité. L'action conjointe de nomination que nous préconisons d'engager avec et auprès des endeuillé $e \cdot s$ connaît des précédents. L'exemple du rapport du Conseil supérieur de travail social publié en 2015 met au jour le désaccord des concernés·e·s sur la désignation usagers (CSTS 
2015, «Refonder le rapport aux personnes "Merci de ne plus nous appeler usagers" »). Dans le cas de terrains sensibles, les hétérodésignations ne vont pas de soi et il incombe aux chercheur·e's de faire apparaître ces désaccords linguistiques.

Dans ce but, nous commencerons par faire l'inventaire des autodésignations utilisées par les concerné·e's. Nous montrerons ensuite que certain'e-s d'entre elles'eux tentent de faire entendre leur voix par une action publique et des demandes de réflexion terminologique puis nous soulignerons l'activité métalinguistique des énonciatrices·teurs dans leur quête d'un désignant adapté à leur situation. Pour conclure, nous présenterons les pistes de prolongement de cette étude.

\section{Des désignations spontanées non consensuelles}

Une série (très variée) de désignations nées spontanément témoigne de l'absence d'une désignation consensuelle. L'absence de consensus se manifeste non seulement au sein de la communauté des parents endeuillés mais aussi entre endeuillé·e·s et non endeuillé e·s, comme en témoignent l'écart entre certaines hétérodésignations (celles que l'on trouve dans le discours médico-juridique ou dans les commentaires internet des pétitions en faveur de la création d'un mot, voir infra) et les désignations proposées par les endeuillé·e's dans leurs témoignages et leurs pétitions, ainsi que les multiples commentaires de linguistique profane évaluant les diverses désignations.

\subsection{Les désignations des enfants décédés : étoile et ange}

Les catégories d'autodésignation et d'hétérodésignation ne suffisent pas à circonscrire le champ de la désignation car elles ne s'appliquent pas aux désignations données par les concernée·e·s aux enfants décédés (ange par exemple) pour lesquels nous parlerons donc de «hétérodésignation située ». Nous portons notre attention sur deux hétérodésignations récurrentes, ange et étoile. Celles-ci sont employées par les endeuillé·e·s (exemples 1 à 3) et sont souvent reprises par les non endeuillé·e·s (exemple 4).

Ex. 1 .

Nous pensons à eux et à leur famille / OCTOBRE / [..] 1 : Grâce et ses parents, Clémentine et Nicolas [...] 4 : Aimé et ses parents, Mireille et Lionel [...]12: Ange et ses parents, Séverine et Jean-Pierre [...] 12 : 2 Petites Étoiles Filantes (Jumeaux) et leurs parents, Christine et Nicolas [...]

(association Petite Émilie, Lettre 19, octobre 2010, p. 8, rubrique « Nous pensons à eux et à leur famille », mis en gras par nous)

Ex. 2.

Les prénoms de nos anges

(titre d'une discussion sur le forum doctissimo, 15 avril 2013)

Ex. 3

Chers petits Anges,

Vous êtes tous réunis là-haut... Nous, vos Mamans, nous sommes aussi réunies, en bas, pour nous aider mutuellement à surmonter votre absence, car vous nous manquez, et l'on vous aimera toujours du plus profond de notre cœur...

Petits Anges, voici toutes vos Mamans

Regardez : nous avons toutes allumés nos bougies pour vous petits anges...

(forum magicmaman, Les mamans des anges, 2 février 2006, mis en gras par nous) 


\section{Ex. 4}

a toutes les mamnange plein de millier de bisous a vous et plien de pensées a vos anges pour ce noel si dur a vos yeux[...]

millier de bisous de [prénom] et sa maman

(forum doctissimo, 22 décembre 2008, mis en gras par nous²)

Dans ce corpus, la métaphore de l'ange ou de l'étoile permet, par l'attribution d'une nouvelle identité, une métamorphose discursive. Il s'agit au sens de Achard-Bayle (2008) de désignations métamorphiques, c'est-à-dire de désignations qui dépassent le stade métaphorique. Selon Guy Achard-Bayle (2008 : 96), le processus de perception métamorphique «met deux objets de discours non coréférentiels en relation avec un seul objet du monde » alors que la saisie métaphorique «met en relation deux référents du monde dans un seul et même espace mental et partant de discours ». Dans l'exemple 1, qui consiste en une liste des enfants décédés publiée mensuellement par l'association Petite Émilie, l'énoncé 2 Petites Étoiles Filantes exprime une métamorphose de l'enfant devenu étoile filante. Ce syntagme nominal lexicalisé permet d'exprimer à la fois que l'enfant est décédé, puisque devenu étoile, et aussi qu'il a peu vécu puisqu'il s'agit d'une étoile filante (la traînée lumineuse de l'étoile filante est éphémère).

Les exemples 2 et 3 présentent le mot ange qui est la désignation la plus partagée. Il ne s'agit pas simplement d'une désignation hypocoristique. Le terme est employé aussi bien comme appellatif dans des discours adressés à l'enfant décédé (exemple 3) que comme désignant générique ainsi que le montrent l'exemple 2 (de nos anges) ou bien encore l'expression lexicalisée carré des anges qui désigne le secteur réservé aux enfants dans les cimetières. Le terme ange figure aussi dans les pseudonymes nécronymes numériques des internautes endeuillés sous la forme [ange + prénom de l'enfant décédé] où ange fonctionne comme un désignateur catégoriel (Ruchon 2017).

L'euphémisation par le recours à la métaphore de l'étoile et de l'ange n'est pas fortuit. Il s'agit de figurer une représentation spatiale et pas seulement de dire la mort. Dans une vision animiste du monde, les parents mettent l'esprit de leur enfant dans l'ange ou l'étoile. L'assimilation à l'étoile ou à l'ange permet de passer d'une métamorphose d'ordre ontologique à la création d'un espace cosmo-discursif où situer les enfants. Comme l'écrit Vinciane Despret (2012 : 39), « les morts posent des problèmes géographiques », il faut «chercher un lieu où les loger, où les abriter, d'où ils peuvent nous interpeller ». Composantes du ciel, les figures de l'ange et de l'étoile permettent de représenter un espace discursif où «loger » les enfants décédés (le ciel). Il offre asile aux esprits des enfants qui parfois n'ont pu avoir de sépulture matérielle. C'est par la métaphore, par le recours à des référents évolutifs (Charolles et Schnedecker 1993, Achard-Bayle 2000) et par le lexique, que s'opèrent la métamorphose de l'enfant et la constitution de son nouvel univers, univers symbolique et cosmique. Ce lieu est un espace relationnel, c'est-à-dire relié aux énonciatrices-teurs, et axiologique car doté de la valeur positive du haut ( $v s$ celle du bas). En effet, dans les représentations, le haut est valorisé, au contraire de la frontalité et de la latéralité (de Chanay et Rémi-Giraud 2002).

Relevant d'une conception animiste, les images de l'ange et de l'étoile montrent que le langage permet de modifier la réalité sans la dénier, ce qui réaffirme le primat du constructivisme langagier. Cette cosmogonie laisse émerger en discours différents modes de vérité, entre nature et culture, sans que les parents puissent être taxés d'irrationalité. Ce lieu réel (le ciel) est aussi un lieu de projection où situer les enfants devenus anges ou étoiles. Le ciel est à la fois topie et utopie. Les deux « régimes de vérité » (Latour 2012) ne s'opposent pas ; au contraire, ils s'associent dans une vision animiste du monde où l'humain et le non-humain se rejoignent.

\subsection{Autodésignations et hétérodésignations des parents d'enfants décédés}


Les parents se désignent par une gamme étendue d'expressions lexicales. On relève en particulier le paradigme lexical autour du mot ange (mamange, papange, parange, tatange) et les lexies composées [parents + qualificatif (orphelins, désenfantés, en deuil)]. Sur les forums de l'internet, les désignations néologiques de type mamange sont reprises par les non endeuillé·e·s. Hors ligne, ces derniers recourent plus aisément à des désignations plus neutres, du type parent en deuil.

\subsubsection{Mamange, papange et parange, un paradigme désignationnel autour de la désignation ange}

Dans l'usage, l'autodésignation mamange est la plus représentée pour combler la lacune lexicale (418 000 résultats le 17 janvier 2020 pour le mot mamange au singulier et 30500 pour mamanges contre 343000 pour parents désenfantés et 7150 pour parents orphelins). Les occurrences sont très nombreuses, aussi bien en nom commun comme désignateur générique qu'en pseudonyme. Diachroniquement, c'est à ange que l'on doit le mot valise mamange ( «maman d'un ange») et ceux qui ont été construits par analogie (papange pour «papa d'un ange », parange pour «parents d'un ange », tatange pour «tante d'un ange $»$ ).

Ex. 5

Alors voilà effectivement avant d'être maman j'ai été mamange. Mamange est un mot que j'aime beaucoup qui signifie «Maman d'un ange». Je trouve ce mot tellement beau. [...] Nous sommes les «paranges» et parents les plus heureux à ce jour mais je n'oublierai jamais mais alors jamais ce que l'on a vécu avant d'avoir nos petites puces.

(forum elleraconte, Témoignage d'une mamange, 1er août 2008, en gras dans le texte)

L'exemple 5 comporte un énoncé métalinguistique profane sur le mot mamange. Il appartient à un article de blog publié en 2008. L'internaute reprendra cet article presque intégralement en 2009 mais supprimera ce passage explicatif, ce qui laisse supposer que le terme est alors entré dans le registre.

Ex. 6

C'est juste ça la vie de mamange... Mais en aucun cas tu n'as à culpabiliser. Tu es la maman de deux petits garçons et même si le monde entier voudrait te faire croire le contraire tu sais au fond de toi où est la vérité.

(forum doctissimo, Vivre après avoir perdu son bébé, 15 janvier 2013, mis en gras par nous)

Ex. 7

C'est ça la vie de mamange. [emoticone qui sourit, emoticone qui pleure]

(forum auféminin, Journée colère, 24 novembre 2010, mis en gras par nous)

Dans les énoncés des exemples 6 et 7, la forme mamange apparaît comme un mot du lexique usuel, en substitut du mot maman, dans une collocation où l'on pourrait s'attendre à trouver le mot maman. Les énoncés C'est juste ça la vie de mamange et C'est ça la vie de mamange, où le mot mamange ne porte aucune marque de modalisation autonymique, n'est souligné par aucun commentaire métalinguistique, montrent à quel point ce néologisme est devenu pour les endeuillées un terme usuel. 


\subsubsection{L'expression parent en deuil, une hétérodésignation}

La désignation parents en deuil est un syntagme répondant aux règles compositionnelles de la langue. En dépit de ce fait, et de sa neutralité axiologique, elle semble peu employée par les parents pour s'autodésigner.

\section{Ex. 8}

oui sa ne ceux fait pas

c honteux de oser dire sa a des parents en deuil quand on est pas dans la situation on ne juge pas (forum doctissimo, Décès de mon fils [initiale prénom], 16 septembre 2008)

Ainsi dans l'exemple 8, la désignation parent en deuil est employée par une internaute n'ayant pas perdu d'enfant. C'est cette désignation neutre, non subjective, que choisissent généralement les intervenant·e·s extérieur·e·s. La langue peut ainsi refléter le degré d'implication des locutrices'teurs en fonction de leurs choix lexicaux par l'opposition entre autodésignations et hétérodésignations (Chachou 2012).

On peut supposer que cette préférence soit due à l'aspect duratif de la forme parent en deuil qui laisse entendre que l'état « en deuil » puisse avoir une fin. Au contraire d'une expression comme parent endeuillé où l'adjectif endeuillé qualifie explicitement le nom qu'il définit sans envisager le début ou la fin de cette caractérisation. En effet, l'énoncé il est resté longtemps en deuil de sa fille est acceptable tandis que il est resté longtemps endeuillé de sa fille est beaucoup plus discutable. Il semblerait ainsi que les personnes non impliquées dans le deuil d'enfant sélectionnent de préférence une désignation à l'aspect non perfectif. La construction $N$ (parent) $+e n+N$ (deuil) décrit un état passager (être en deuil, en colère, en panne, en fuite, etc.) attribuable à une chose ou un individu, faisant suite à un événement (voir ce que dit Leeman 1995 du caractère résultatif de $e n+N$ ). La transformation opérée suite à cet événement ne serait alors que temporaire (être en larmes suite à un événement triste, être en grève suite à une mesure patronale). Même une expression comme être en pente fait référence à un état passager : une route en pente est la continuité d'une route qui pouvait être plane et qui de plus selon le point de vue énonciatif peut aussi qualifiée de en côte. Le caractère temporaire de en $+N$ pourrait d'ailleurs expliquer que l'énoncé *en mort ne soit pas usité alors que l'énoncé en vie, évoquant un état temporaire qui implique une fin, soit acceptable.

\subsubsection{Aux origines de la lacune lexicale française, la lexie composée parent orphelin}

La lexie parents orphelins permet de créer une synthèse disjonctive par une apparente incohérence sémantique entre les mots parent et orphelin. Cela n'a pourtant pas toujours été le cas. Cette lexie s'appuie sur les conditions d'emploi de l'adjectif grec orphanè qui s'appliquait à différents contextes de deuil, quel que soit le lien entretenu avec la personne décédée. Le mot d'origine grecque orphelin a perdu le sens général de privé de (son enfant, son neveu, ses grands-parents, etc.). Il s'est spécialisé et désigne exclusivement l'enfant privé de ses parents. L'Église, mais aussi l'État, pourraient avoir eu intérêt à endiguer les débordements pathétiques relatifs à la mort de l'enfant (Cazal 2009a, 2009b). On trouve cette expression dans le titre d'un ouvrage témoignage (exemple 9) et dans le nom d'une association québécoise (exemple 10) :

Ex. 9

Parents orphelins. Témoignages de parents à la suite du décès de leur enfant (titre d'ouvrage, édition Fafard Sindon, 1990) 
Ex. 10

Fondée en 2008, l'association Parents Orphelins (Canada) a mis sur pied une campagne de sensibilisation entourant la date du 15 octobre, en référence aux différentes activités et célébrations, ayant lieux dans plusieurs pays à cette date.

(association Petite Émilie, lettre 27, octobre 2012, article « Journée internationale de sensibilisation au deuil périnatal», p. 6)

La désignation parents orphelins n'a pas été sélectionnée par les parents pour un emploi usuel (en France du moins car elle semble se développer en Belgique et au Québec). C'est peut-être parce qu'elle fonctionne mieux comme hétérodésignation permettant de désigner plus facilement un groupe qu'un individu, parent s'employant usuellement plus souvent au pluriel qu'au singulier, ou peut-être aussi parce que les concerné·e·s s'identifient plus aisément dans une désignation non composée, comme mamange. Pourquoi mamanges estil plus fréquent sur l'internet que les expressions concurrentes, avec 418000 résultats le 17 janvier 2020 pour le mot mamange au singulier et 30500 pour mamanges ? Un chiffre en ascension puisqu'on comptait 147000 résultats sur le moteur de recherche de Google le 21 février 2015 pour mamange. Et pourquoi l'emporte-t-il sur son équivalent synaptique maman d'un ange ? L'échec discursif d'expressions désignantes telles que parents endeuillés (30 000 résultats en 2015, 32300 en 2020), parents en deuil (45 900 résultats, 48500 en 2020), voire de maman d'un ange (38 000 résultats, 99900 en 2020), et la fréquence d'utilisation de mamange laissent penser que la catégorisation «maman d'un enfant décédé » exigerait un item lexical unique. S'appuyant sur l'exemple de la dénomination ophtalmologue et de sa correspondance désignante médecin spécialiste des yeux, Georges Kleiber constate en 2001 que seul ophtalmologue constitue une "unité formelle », tandis que la séquence médecin spécialiste des yeux n'est pas une «unité formelle, préconstruite », et donc que seule la dénomination ophtalmologue permet l'aspect catégoriel de la dénomination (Kleiber 2001 : 36-37). Sur le plan formel, la catégorie ne pourrait être contenue que par un item lexical unique, ou éventuellement un mot composé, et non par une paraphrase ou une glose explicative. Cela expliquerait le succès d'un mot valise mamange sur l'expression syntagmatique maman d'un ange. Ainsi, au-delà de la métaphore originelle, le mot ange a-t-il ouvert une catégorie là où ont failli des séquences descriptives telles que nourrisson décédé de mort subite ou bébé décédé in utero, ou enfant décédé précocement, précisément parce qu'elles ne sont pas, selon les termes de Georges Kleiber, des unités formelles (Kleiber 2001 : 37).

\subsubsection{Le syntagme néologique parent désenfanté}

Ce syntagme composé d'un nom catégoriel (parent) et du participe passé adjectivé néologique (désenfanté) semble gagner du terrain en tant que hétérodésignation, en particulier à Bruxelles. En tant qu'auto-désignation, parent désenfanté apparaît dans des emplois institutionnels et très peu dans des échanges interactionnels, par exemple dans le nom d'une association de parents :

Ex. 11 


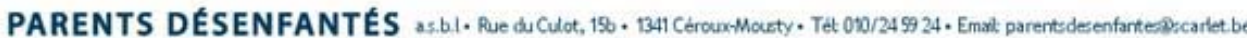

\section{PARENTS DESENFANTES asbin Redaculat,

\begin{tabular}{|l|l|l|l|l|l|l|l|l|}
\hline Historique & Notre accompagnement & Contacts & Agenda & Témolgnages & Blbllothdque & Toxtes & Vos commentalres & Llens \\
\hline
\end{tabular}

\section{HISTORIQUE}

\section{Notre raison d'être}

La mort d'un enfant plonge les parents et les proches dans une souffrance qui peut déstabiliser, faire perdre tout repère, ébranler jusqu'au sens même de la vie et conduire ả une solitude pouvant couper tout lien social. C'est lidentité même de la personne qui est menacée: elle en souffre physiquement, psychiquement et spirituellement.

«Plus rien n'est comme avant $»$.

Ecoute, solidarité, partage, espoir, voilà ce dont ont besoin les parents qui perdent un enfant. Dans les mois qui suivent le décès, il peut devenir de plus en plus difficile de trouver quelqu'un à qui parler et de bénéficier d'une écoute en profondeur.

Dans cet esprit, «parents désenfantés asbl» organise depuis 30 ans des rencontres entre parents endeuillés, pour leur permettre de parler et de s'écouter dans un climat respectueux et chaleureux. Ce que la plupart des parents ressentent en venant chez nous, c'est d'avoir été compris, et de se sentir moins seuis face à cette épreuve.

«Pour que quelqu'un parle, II faut qu'un autre écoute».

Des parents eux-mêmes touchés par le deuil d'un enfant se proposent bénévolement pour écouter d'autres parents endeuillés, et pour leur offrir une présence et un soutien chaleureux. Ils sont formés à l'écoute et a l'animation de groupes, participent à une supervision régulière et bénéficient d'une formation permanente.

Lidée est née en 1981, lorsque deux familles éprouvées par la mort de leur enfant ont compris limportance de trouver, pour elles-mêmes, un lieu de parole, d'écoute et de soutien. Elles organisèrent des groupes de parents, grand-parents, frères et soeurs endeuillés. Ainsi fut créée l'association «Parents désenfantés», qui se constitua en asbl en 1997.

(site de l'association belge Parents endeuillés, créée en 1997)

C'est la collocation parents endeuillés qui est employée dans le texte de présentation de cette association belge, et non parents désenfantés. Parmi les reprises anaphoriques, on trouve aussi la périphrase Des parents eux-mêmes touchés par le deuil d'un enfant.

\section{Ex. 12}

Re : désormais je suis une mamange.../Une année passée sans Toi mon Ange... [...] Une pensée particulière à tous les parents «*désenfantés*» pour qui (je suppose) chaque nouvelle année n'est pas une étape facile à passer !

(forum magrossesse.com, Désormais je suis une mamange, 1er janvier 2008)

Dans l'exemple 12, l'expression parents désenfantés est coréférente avec la forme mamange. Cependant l'agent énonciatif a doublement isolé du reste de l'énoncé le mot désenfanté par des guillemets et par des astérisques. Or la forme mamange, bien que néologique elle aussi, n'est pas modalisée. Pourtant, mamange pourrait être considéré bien plus aux périphéries du système que parents désenfantés, qui est relativement motivé sur le plan formel : cette lexie composée se constitue du mot parent et du verbe enfanter auquel est adjoint le préfixe dé(s)- qui signifie la séparation, la cessation. Une expression comme parent désenfanté est conforme aux règles morphosyntaxiques (nom commun + participe passé adjectivé préfixé) de la langue française. Bien que non lexicalisé, le terme désenfanté n'est pas à proprement parler néologique. Il n'a pas été inventé par les internautes mais par André Chouraqui afin de traduire la Bible de l'hébreu biblique en français (Cazal 2009b : 115). Voici le texte qui figure dans la Genèse : 
Él Shadaï vous donnera des matrices en face de l'homme. Il vous enverra votre autre frère et Biniamîn. Et moi, le désenfanté, je resterai désenfanté. (Genèse 43:14).

En dépit de cette origine biblique, qui pourrait être avancé comme argument légitimant, en dépit d'une conformité morphosyntaxique au code linguistique et d'une relative motivation, cette forme composée est peu plébiscitée, du moins en France.

La diversité des désignations et l'écart entre hétérodésignation et autodésignation soulignent la dimension éthique et politique des faits de nomination dans le cadre du deuil de l'enfant. Comment nommer le mort-né et ses parents? Les endeuillé'e-s s'efforcent de répondre à cette question en recourant à des pétitions citoyennes.

\section{Faire entendre sa voix. Propositions lexicales par les endeuillé'e-s et démarches citoyennes de nomination.}

Nous envisageons la vulnérabilité des parents endeuillés comme un processus dynamique (Ghliss, Paveau et Ruchon 2019) et non comme un état d'impuissance, rejoignant en cela les réflexions du sociologue Jean-Paul Payet pour qui la faiblesse est un processus permettant l'émergence de capacités (Payet et Laforgue 2008, Payet 2011). Les discours métalinguistiques des parents endeuillés mettent en exergue cette faculté. Leurs analyses linguistiques populaires (Achard-Bayle et Paveau 2008) les amènent à une position d'expert sur un plan médical (expertise du lexique médical) et juridique (expertise des textes juridiques et de leurs subtilités linguistiques) (Ruchon 2015 : 37-76). Plus encore, ces discours soulignent la lacune lexicale : il n'y a pas de mot pour désigner celui ou celle qui a perdu son enfant. En 1966, Henry Hoenigswald insistait déjà sur la nécessité de s'intéresser à la «folk linguistics» et aux propos relatifs au langage (Hoenigswald 1966 : 20), comme le rappelle Jean-Claude Beacco (2004) dans l'introduction du numéro de Langages consacré aux représentations métalinguistiques ordinaires. Les énonciatrices·teurs, qui ont acquis une parole d'expert, expriment des revendications lexicales pour pallier cette lacune lexicale. Dans ce corpus, trois exemples de pétition en attestent. Les agents énonciatifs ne se contentent pas du répertoire lexical existant et vont soumettre aux instances institutionnelles des formes inédites telles que parents désenfantés ou parange.

\subsection{Un exemple de pétition citoyenne pour ajouter le mot paranges au dictionnaire français Larousse}

L'exemple suivant concerne le mot paranges qu'une internaute endeuillée souhaite faire entrer dans le dictionnaire Larousse, en utilisant le media citoyen change.org :

Ex. 13 


\section{Larousse : un nom dans le dictionnaire pour les paranges, parents ayant perdu un enfant.}
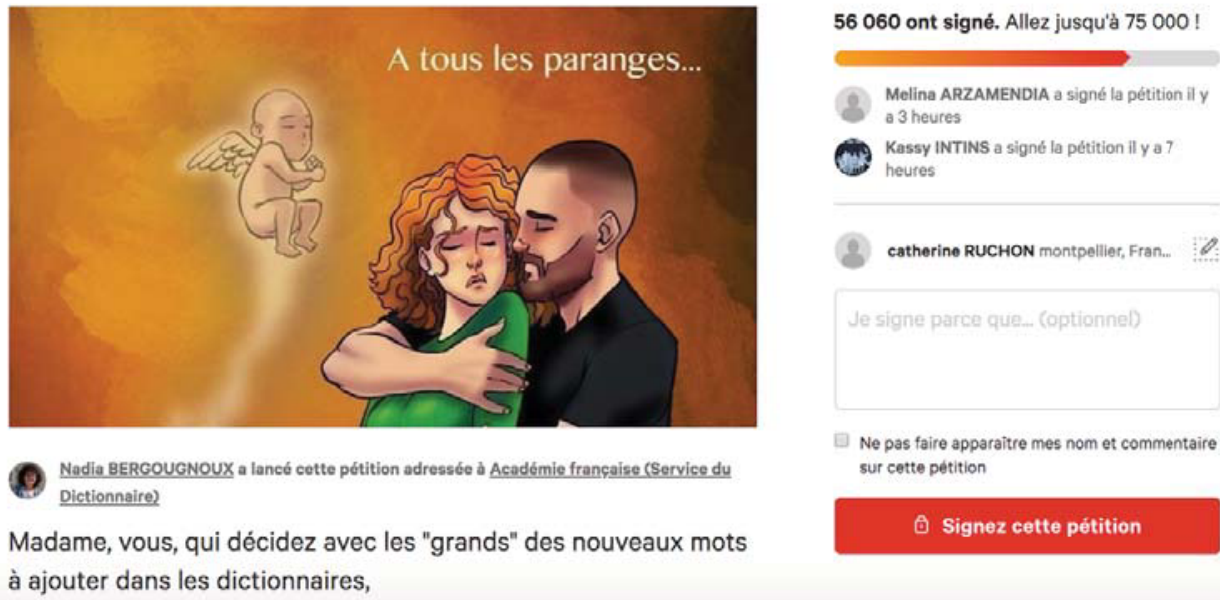

Madame, vous, qui décidez avec les "grands" des nouveaux mots à ajouter dans les dictionnaires,

Je suis une mamange, mot inventé par quelqu'un sans doute qui a subi ce drame... J'ai créé un groupe sur Facebook qui accueille les paranges. Nous sommes plus de 800 à nous soutenir, d'autres groupes existent, d'autres associations...Parce que ce deuil est tabou ! parce que nous ne pouvons en parler qu'entre-nous ! parce que nous subissons, de ce fait, un double deuil, la perte de notre enfant et le silence autour de ce drame, cette reconnaissance, par un mot qui nous qualifiera, est très importante pour nous !

Notre demande englobe, non seulement les parents ayant subi un deuil périnatal, mais aussi tous les parents ayant perdu un enfant, quel que soit l'âge de son décès.

Nous sommes des milliers, en France et ailleurs à avoir perdu un ou plusieurs enfants... que ce soit avant, pendant ou après l'accouchement.. et même longtemps après...

Quand une femme perd son mari, elle est veuve, quand un enfant perd un parent, il est orphelin...

Quand nous perdons nos enfants, que sommes-nous?

Nous sommes des PARANGES! Parents d'Anges

Le mot « ange », dont la définition du dictionnaire est : « Être céleste intermédiaire entre Dieu et l'homme. Personne qui semble douée de toutes les perfections. Personne qui possède au plus haut degré une qualité physique ou morale : C'est un ange de beauté, de douceur. Terme d'affection : Mon ange », conviendrait à tous, croyants et non croyants. Il est d'ailleurs, aujourd'hui utilisé par les PARANGES (comme "mamange » pour une femme, et «papange, pour un homme).

Le deuil d'un enfant, qu'il soit né ou pas est une épreuve indicible et cruelle. pour faire une part du chemin notre deuil, nous aimerions être reconnu(e)s.

Il existe aujourd'hui de nouveaux mots : "googliser", "poilade", "selfie" "zlataner" et tant d'autres !

Aujourd'hui, une femme sur quatre perd son enfant avant ou juste après la naissance... des parents perdent leur enfant et n'ont pas de nom à donner... alors, s'il vous plait... réfléchissez et agissez ! 
(change.org, pétition adressée à Larousse pour faire entrer le mot parange dans le dictionnaire lancée en 2016 par une mère endeuillée, https://www.change.org/p/larousse-un-nom-dans-ledictionnaire-pour-les-paranges-parents-ayant-perdu-unenfant?fbclid=IwAR3vQ0QUA3ie6jkHiR3NwA1picDApE1f-RGL8VJexwwHioBS kLXEc-x0Lo, consulté le 16 janvier 2020, mis en gras par nous)

Dans l'énoncé de cette pétition créée le 27 février 2016, l'internaute autrice qui se qualifie de mamange s'appuie aussi sur la lexicologie pour légitimer sa demande (Le mot " ange », dont la définition du dictionnaire est). À cet argument d'autorité s'ajoute ceux de l'usage (Il est d'ailleurs, aujourd'hui utilisé par les PARANGES) et de l'exemple (Il existe aujourd'hui de nouveaux mots : "googliser", "poilade", "selfie" "zlataner"). Selon le quotidien Le Figaro, Larousse aurait ajouté le terme parange dans sa base de néologie ${ }^{3}$.

Cependant la politique linguistique en matière de néologie est attentiste, elle vient entériner des termes dont l'usage est attesté. Lorsqu'elle propose, c'est en réaction à des termes jugés non conformes entrant dans l'usage (courriel/mail par exemple). Ainsi que le soulignent les auteurs de Un prêt à parler : le dictionnaire : "Les mots arrivent dans le dictionnaire, à travers des usages codés, répertoriés. C'est pourquoi, ils ne signifient que par référence à des assemblages archivés. » (Collinot et Mazière 1997 : 87).

\subsection{Un exemple de demande à l'Académie française pour parent désenfanté}

L'exemple ci-dessous concerne la pétition lancée par une endeuillée pour promouvoir l'entrée de l'adjectif désenfanté dans le dictionnaire de la langue française :

Ex. 14

Après deux ouvrages sur la disparition de son petit Lucas, Francine Vervick de Travecy entend proposer à l'Académie française de faire entrer l'adjectif « Désenfanté » dans le dictionnaire. Sa définition? La perte d'un enfant par ses parents. [...] « J'ai entrepris cette démarche voici une quinzaine de jours seulement quand j'ai entendu les nouveaux mots qui allaient entrer dans le dictionnaire. Des termes de la société moderne dont certains tiendraient presque du verland des jeunes. Bref, c'est sans commune mesure avec l'adjectif désenfanté, lequel s'il est retenu, permettrait de mettre un nom sur une situation plus répandue qu'on ne le pense et devrait faire bouger davantage les pouvoirs publics. Après avoir fait jouer mon carnet d'adresse sur Internet, j'ai déjà reçu une centaine de lettres et mails pour soutenir ma démarche. Notamment de Belgique, du Canada et des États-Unis. Car la langue de Shakespeare rencontre ce même écueil », explique Francine. Pour l'heure, Francine constitue son dossier. En s'intéressant aux démarches similaires entreprises par d'autres dans un passé proche, elle a découvert qu'il fallait démontrer à l'Académie française qu'il existe un réel manque et que ce terme est déjà usité dans le langage courant. «Personnellement, j'utilise depuis le début l'adjectif « désenfanté » sur mon site Internet, ainsi que de nombreux autres forums... Outre un courrier argumenté présentant les raisons de ma démarche, j'entends y glisser une pétition et des courriers et mails de soutien. Car il est très difficile, juridiquement parlant, de faire valider une pétition dont les signatures sont recueillies sur la toile ».

(journal L'union, 26 septembre 2007)

Le discours rapporté dans l'exemple 14 porte les caractéristiques d'un énoncé puriste tel que le définit Laurence Rosier (2004) :

Il [le discours puriste] se caractérise par une forte axiologisation performative (ce qui se dit, ce qui ne se dit pas) qui rejoint le bon usage et entend respecter une stricte économie des échanges linguistiques, où on évalue celui qui parle selon sa maîtrise de 
la langue, sous l'angle de la richesse lexicale et de la correction grammaticale. Il relève du discours normé tel que le définit Berrendonner : instrument de pouvoir sur la parole d'autrui se présentant comme légitime et autorisé, il « masque sa visée conative en projet constatif en usant d'arguments esthétiques (beau/laid), politiques (langue de la liberté), linguistiques (clarté de la langue), métaphoriques (langue en bonne santé). En outre, il cultive la nostalgie par l'idéalisation de pratiques antérieures érigées en modèles désormais inaccessibles (Rosier 2004 : 69)

L'exemple 14 présente de nombreux commentaires appréciatifs sur la langue à connotation sociologique (termes de la société moderne, verlan des jeunes) et particulièrement axiologiques sur la langue, comme en témoigne la référence à la langue de Shakespeare, sélectionnée de préférence à la désignation non axiologique anglais, Shakespeare représentant métonymiquement les grands auteurs de littérature. L'agent énonciatif pratique une sociolinguistique profane par un classement des manières de parler (Rosier 2008 : 58-63 en particulier). Le commentaire du mot termes (« de la société moderne dont certains tiendraient presque du verland des jeunes ») sous-tend implicitement qu'il y aurait deux types de termes : l'un soumis aux modes langagières de la société moderne (le complément du nom de la société moderne apportant cette restriction), l'autre fonctionnel et nécessaire (car permettant de «mettre un nom sur une situation plus répandue qu'on ne le pense » et de « faire bouger davantage les pouvoirs publics »). Ces commentaires métalinguistiques vont en faveur d'une soumission à la norme. L'agent énonciatif souhaite disposer d'une expression adaptée à sa situation, mais ce souhait est conditionné à une "mise en conformité » linguistique par l'entrée dans le dictionnaire. En outre, ce commentaire prône une adéquation entre la langue et le monde (mettre un nom sur une situation plus répandue qu'on ne le pense).

Par la suite, le 10 juin 2014, l'internaute a aussi publié sur WikiLF l'entrée désenfanté. Elle y présente notamment un corpus des différents emplois :

Ex. 15

Le principe de la compositionnalité des dérivés est respecté. [...] Nous avons donc affaire à un néologisme de bon aloi qui ne heurtera pas la conscience linguistique des lettrés et dont l'introduction dans les dictionnaires serait légitime.

(site wikiLF.culture, Désenfanté, 10 juin 2014)

L'article ci-dessus (exemple 15) met en avant l'argument morphosyntaxique. Les nombreux commentaires de l'article révèlent d'ailleurs le souci du respect de la morphosyntaxe quand il s'agit de néologie. Les démarches de folk terminologie des parents endeuillés rendent compte du souci de conformité linguistique.

\subsection{Un exemple de pétition citoyenne : « Ça ne se dit pas »}

L'exemple ci-dessous est une pétition citoyenne mise en ligne en mai 2014 par une mère endeuillée :

Ex. 16

Ça ne se dit pas

IL MANQUE UN MOT DANS LA LANGUE FRANÇAISE. COMMENT POUVONSNOUS RÉFLÉCHIR SUR CETTE ABSENCE DE MOT ET AGIR ENSEMBLE POUR Y REMÉDIER ?

Il manque un mot dans la langue française. Chaque Français doit pouvoir utiliser sa langue dans les divers domaines de sa vie quotidienne. Ce droit n'est pas respecté pour le 
parent qui a perdu son enfant et qui doit répondre de sa situation familiale notamment sur les documents administratifs : Avez vous des enfants ? Combien avez vous d'enfants? Le parent d'un enfant décédé est toujours le père ou la mère de cet enfant, alors comment doit-il répondre à ces questions?

Monsieur Jean Paul Delevoye président du Conseil Economique, Social et Environnemental, vous avez le pouvoir de traiter d'une question particulière via une autosaisine; c'est pourquoi nous vous sollicitons par voie de pétition citoyenne à encourager une réflexion sur cette absence d'un mot dans notre langue.

Demander au parent de répondre de son statut familial dans la vie quotidienne et sur certains documents administratifs mais ne pas lui permettre de répondre de l'enfant in absentia

c'est l'inciter à renier la mémoire de cet enfant

c'est l'obliger à évoquer la mort pour parler de l'enfant

c'est le condamner à l'isolement par peur du «comment le dire»

c'est amputer le parent de l'amour que l'enfant a fait grandir en lui.

Nous signons cette pétition afin de soutenir et d'encourager cette cause juste et profondément humaine.

(pétition Ça ne se dit pas, mise en ligne sur internet en mai 2014,

http://caneseditpas.wesign.it, consulté le 22 février 2015)

Cette pétition a pour objet la création d'un mot permettant de désigner le parent qui a perdu son enfant, le parent d'un enfant décédé. La revendication - à la fois politique, sociale et discursive - contre une oppression marquée par la modalité déontique (doit, obliger, permettre), s'exprime par le lexique juridique (droit, condamner), mais aussi par un jeu de mots sur la locution Ça ne se dit pas, habituellement employée à propos des « mésusages » de la langue et pris ici dans son sens littéral : être parent d'un enfant mort ne se dit pas. Surtout, ce jeu de mot reflète la soumission linguistique à la norme en vigueur : certaines choses se disent, d'autres non. Au-delà de l'appel aux valeurs dans les exemples 13 (épreuve indicible et cruelle) et 16 (amour, mémoire, cause juste et profondément humaine), les exemples 14, 15 et 16 témoignent du désir de conformité à la norme linguistique. Soumis à une oppression linguistique, exprimée notamment dans l'exemple 14 avec les mots décidez et grands (Madame, vous, qui décidez avec les "grands" des nouveaux mots à ajouter dans les dictionnaires), les agents responsables de ces actions souhaitent un terme reconnu et cautionné par le dictionnaire.

Les exemples de pétition précédents (exemples 13,14,16) illustrent deux procédés d'action sur la langue : l'un veut entériner un terme préexistant (parange dans l'exemple 13, et désenfanté dans l'exemple 14, mot dont les agents soulignent l'antériorité discursive), l'autre veut engager une réflexion sur la création d'un terme ex nihilo (exemple 16). C'est la première des actions qui jusqu'à présent est conforme aux us et coutumes terminologiques, soumises à une norme externe de « décideurs intellectuels pris dans des positions idéologiques sur la langue » (Collinot et Mazière 1997 : 208). La seconde mérite cependant réflexion. Ne signale-t-elle pas une modification du comportement de la du locutrice-teur qui devient un·e produser (Bruns 2008) de la langue, c'est-à-dire un participant actif du système linguistique, à la fois usager et producteur de discours ? Dans les deux cas, le fait qu'une addition lexicale soit proposée par des usagers profanes et non par des instances officielles est notable. C'est un événement, la mort d'un enfant, qui a suscité ces démarches :

Phénomène de discours, l'événement peut se comprendre comme l'irruption d'une énonciation qui viendrait déstabiliser des positivités établies et mettre en place, dans la plus grande discrétion, un réseau de nouveaux rapports entre les discours. Événement et énonciation ont partie liée. (Collinot et Mazière 1997 : 210) 
Les sujets énonciateurs prennent la langue comme objet de discours dans une activité métalinguistique dont cette étude a révélé de nombreuses manifestations.

La douleur semble donner des droits discursifs. Cependant les discours du locutor dolorosus laissent apparaître une relative soumission aux normes linguistiques, ce que soulignent les modifications opérées sur le texte de la pétition au fil du temps. L'autrice a en effet étayé son argumentation par des références étymologiques :

Ex. 17

\section{Ça ne se dit pas}

IL MANQUE UN MOT DANS LA LANGUE FRANÇAISE. COMMENT POUVONSNOUS RÉFLÉCHIR SUR CETTE ABSENCE DE MOT ET AGIR ENSEMBLE POUR Y REMÉDIER ?

Le grec ancien et le latin disposaient d'un mot

Le grec ancien et le latin disposaient d'un mot à partir de orphanus (en latin), orphanos (en grec) pour désigner aussi bien le parent qui avait perdu un enfant que l'enfant qui avait perdu un parent. L'adjectif orbus que Félix Gaffiot traduit par «privé d'un membre de sa famille» se disait aussi bien pour un parent que pour un enfant. Ce mot a permis en latin chrétien l'expression mater orba mais orbus a disparu au moment du passage aux langues romanes.

Pour apparaitre dans le dictionnaire un mot doit être déjà ancré dans la langue, il faut donc le faire circuler - lui donner une identité et un visage pour lui accorder une place dans le langage. C'est pourquoi il est important de susciter un mouvement populaire sur cette question de nommer. (pétition Ça ne se dit pas, mise en ligne sur internet en mai 2014, http://caneseditpas.wesign.it, consulté le 20 décembre 2019)

Cependant, même si les énonciatrices ne s'affranchissent pas de la norme lexicographique, leurs actions manifestent une relative autonomie qui répond à la catégorie d'acteur faible (Payet et Laforgue 2008) et s'inscrit dans ce que nous appelons les dynamiques de la vulnérabilité (Ghliss, Paveau et Ruchon 2019).

La lacune lexicale, qui passait encore inaperçue dans les années 1990, moment discursif où commencent à se multiplier les témoignages des parents endeuillés, semble désormais évidente à tou·te·s les endeuillé·e·s. Le texte de présentation de l'ouvrage Orphelins d'un enfant l'évoque explicitement :

Ex. 18

On dit «veuve » ou « veuf » lorsqu'on a perdu son conjoint, « orphelin » lorsqu'on a perdu ses parents, mais il n'existe pas de mot pour désigner les parents qui ont perdu un enfant. Cécile et Benoît sont donc " orphelins » de leur fils, Vianney et Henry de leur frère de sang, les Dominicains de leur frère en religion. Vincent avait trente ans lorsqu'il mourut : il venait de faire «profession solennelle » dans l'ordre des Frères prêcheurs, les Dominicains. Quand Cécile, sa mère, prit des notes dans un petit carnet qui devint un journal relatant ce qu'elle vivait auprès de son fils, alors en phase terminale d'un cancer du poumon, elle était loin de se douter que cela aboutirait à l'écriture de ce livre. Sa route devint une véritable quête spirituelle pour comprendre ce qui avait permis à son fils de passer si vite, sans fléchir, " de la mort à la Vie ». Après de solides études, Vincent avait fait le choix de la vie religieuse pour transmettre sa foi. Comment a-t-il accepté sa mort sans révolte ? Comment sa mère, si passionnément engagée dans sa maternité, peut-elle affirmer que, malgré tout, la vie est magnifique ? Se voulant à la fois un témoignage et un réconfort pour ceux qui, comme elle et Benoît, sont des mères et des pères sans nom, « Orphelins d'un enfant » tente de répondre à ces questions. 
(éditions du cerf, texte éditorial de présentation de l'ouvrage Orphelins d'un enfant de Cécile Paris, 2013, https://www.editionsducerf.fr/librairie/livre/6776/orphelins-d-unenfant, consulté le 16 janvier 2020)

L'énoncé éditorial de l'exemple 18 focalise sur la lacune lexicale en commençant non par le résumé de l'ouvrage mais par le constat de la lacune lexicale (il n'existe pas de mot pour désigner les parents qui ont perdu un enfant). Le titre Orphelins d'un enfant pointe aussi d'emblée la lacune lexicale en recourant au terme orphelin mais sans l'assumer comme désignant à part entière. La présence d'un déterminatif (d'un enfant) fait écho à l'origine étymologique de orphelin qui pouvait concerner les parents ayant perdu leur enfant et non exclusivement les enfants ayant perdu leurs parents comme c'est devenu l'usage. Cette dépendance étymologique s'associe à une dépendance syntaxique et bloque l'emploi autonome de orphelin qui ne peut ainsi constituer une « unité formelle » (Kleiber 2001 : 3637) ni fonctionner comme désignant catégoriel.

Bien qu'elle ne cesse d'être soulignée dans des pétitions ou des interviews d'autrices. teurs comme celle accordée par l'autrice de l'exemple 18 (en ligne sur https://www.youtube.com/watch?v=Vs-CNilJCMo, consulté le 16 janvier 2020), la lacune lexicale n'a toujours pas été comblée. L'échec des démarches citoyennes s'explique en partie par la difficulté de trouver une désignation pouvant aussi bien jouer le rôle d'autodésignation et d'hétérodésignation, comme en attestent les métadiscours linguistiques énoncés par les concernés et les non concernés étudiés dans la partie suivante. Cet échec est aussi très éclairant sur l'invisibilité d'une frange de la population, les parents endeuillés. Le prisme de l'intersectionnalité «donne à voir les dynamiques constitutives du pouvoir invisibilisées par des logiques discursives» (Kocadost 2017, en ligne) mais aussi plus spécifiquement les enjeux de la nomination.

\section{Métadiscours et jugements linguistiques des endeuillé·e·s sur ces désignations}

Les exemples précédents montrent que les endeuillé·e·s théorisent à partir de leur expérience personnelle, acquérant ainsi un statut d'expert. Par un ajustement situationnel des mots, elles·ils opèrent une resignification thérapeutique des termes considérés comme blessants ou posant problème, par exemple en remettant en question le terme ange pour son trait/non incarné/ et en refusant toute assimilation référentielle avec leur enfant (sur les vertus antalgiques du discours, voir Ruchon 2015). L'expérience douloureuse du deuil les a amenéee's à des pratiques d'analyse linguistique populaire, notamment lexicographique (avec des recherches étymologiques et l'identification des désignations latines et grecque (mater orba, mater dolorosa, orphanè). On observe aussi une prise de conscience de la question du point de vue par les endeuillée·s (du type « je suis / ne suis pas papange / mamange $»+$ commentaire).

\subsection{Des désignations contestées}

L'exemple ci-dessous pourrait laisser supposer que le processus de lexicalisation du paradigme lexical mamange/enfange/papange/parange touche à son terme et que la métaphore de l'ange est unanimement acceptée :

Ex. 19

mamange \ma.ma $\tilde{z} \backslash$ féminin

1. Mère ayant perdu un enfant durant la grossesse ou après la maternité. 
- C'est vrai que l'on a souvent des post de mamange mais rare sont ce de papange.- (forum.magicmaman.com, Un papange qui a souffert, mais qui souhaite aider aujourd'hui!!!, 2008.)

Voir aussi

$$
\begin{aligned}
& \circ \text { enfange } \\
& \circ \text { papange } \\
& \circ \text { parange }
\end{aligned}
$$

(Wiktionnaire, https://fr.wiktionary.org/wiki/mamange, consulté le 16 janvier 2020)

La diffusion de ces formes ne va cependant pas sans contestation comme nous allons le constater. On trouve dans le discours des concernées's de nombreuses réticences linguistiques, comme ici sur la forme mamange :

Ex. 20

Pierre : un ange ?

Certaines personnes aiment dire que ce petit bébé devient un ange. Certaines mamans se surnomment « mamanges ». Personnellement, je n'aime pas utiliser cette expression. Un ange, dans l'imaginaire collectif, est une créature mi-divine et / ou mi-humaine. Souvent on dit que les anges n'ont pas de sexe. Or le décès d'un tout petit qui meure avant de naître est un événement tellement tabou que sa réalité même peut être mise à rude épreuve. Le terme d'ange, malgré sa douceur et la tendresse qui s'y rattache, accentue un peu ce côté irréel qui fait sourir de nombreux parents. Si une tante décède on ne dit pas d'elle qu'elle devient un ange ! Alors pourquoi le faire pour un bébé décédé in utero ? N'est-il pas un membre à part entière de la famille ? Pour l'entourage, une mamange n'est pas tout à fait une vraie maman, donc, si on n'est pas une vraie maman, ce n'était pas un vrai bébé, et il n'y a donc pas besoin de le pleurer autant puisque ce n'était pas un bébé ! (ouvrage témoignage numérique d'une mère endeuillée, Nathalie Z, L'un sans l'autre, 2008, p. 140)

Les parents requalifient à de nombreuses reprises les termes employés dans le contexte du deuil périnatal et de l'infertilité. C'est le cas dans l'énoncé ci-dessus où l'agent énonciatif réfute l'emploi des mots ange et mamange usuel dans la communauté des parents endeuillés. La contestation de l'emploi du mot ange est tout d'abord marquée par la modalité interrogative (un ange ?). Cette contestation de la requalification de l'enfant en ange souligne le caractère usuel de la métaphore dans le contexte du deuil périnatal. L'auteure de l'énoncé suivant exprime aussi son refus de valider l'emploi du mot ange :

\section{Ex. 21}

Un ange ! Ce n'est pas un ange que je veux, c'est ma petite fille. Et qu'est-ce qu'une enfant morte peut avoir de beau ? C'est effrayant !

(ouvrage témoignage numérique d'une mère endeuillée, Caroline Paquin, La chambre vide, 2005, p. 27)

L'énoncé ci-dessus montre le refus d'assimilation référentielle. Pour cette auteure, il y a bien deux référents dont elle récuse le lien, notamment par les déterminants ma et un, le premier créant un lien entre l'agent énonciatif et l'enfant, le second référant à une entité indéterminé.

La pétition pour faire entrer le terme paranges dans le dictionnaire (exemple 13) soulève elle aussi des commentaires très critiques : 


\section{Ex. 22}

Je suis contre!

Contre pour plusieurs raisons.

Je suis moi aussi une maman dont l'enfant est mort.

J'ai milité il y a quelques années pour que ces enfants aient une reconnaissance légale, soient inscrits dans le livret de famille, aient droit à un enterrement digne de ce nom pour ceux qui le souhaitent mais je refuse que ce terme soit inscrit dans le dictionnaire !

Ce terme ou un autre, là n'est pas tant le problème.

Je le refuse car je suis maman avant tout et non pas maman d'un enfant perdu....

Non mon enfant n'est pas perdu, il est mort point mais je suis et reste une maman, certes différente mais c'est ainsi.

Et puis, comment nommé l'innommable?

(Facebook de Change.org, 2017, commentaire de la pétition en faveur du mot paranges)

\section{Ex. 23}

Les parents auteurs d'infanticides seront aussi nommés ainsi ? Et pourquoi le suffixe "anges"? Incompréhensible cette pétition ! Y en a une en ce moment pour sauver le droit du travail si vous voulez.

(Facebook de Change.org, 2017, commentaire de la pétition en faveur du mot paranges)

\section{Ex. 24}

Un par'ange c'est pour les parents croyants et pour les autres parents ?

(Facebook de Change.org, 2017, commentaire de la pétition en faveur du mot paranges)

Ces trois commentaires (exemples 22 à 24) soulèvent différents points :

- la resignification du mot maman (je suis maman avant tout et non pas maman d'un enfant perdu). Les parents manifestent souvent dans leurs témoignages la nécessité d'un réajustement lexical de ce type à propos des termes maman, mère ou parent.

- la non coïncidence interlocutive et la non coïncidence entre le mot et la chose (AuthierRevuz 1990).

- la connotation religieuse du mot ange. Ces commentaires métalinguistiques montrent que la désignation ange ne va pas de soi pour l'ensemble de la communauté. En particulier, elle ne fait plus consensus dès lors qu'elle est mise sur la scène publique (via les pétitions citoyennes). Notre expérience personnelle nous a aussi montré qu'elle n'allait pas non plus de soi pour les personnes non concernées, en partie du fait de la connotation religieuse attachée au mot ange. Ces dissensions sur un certain « ressenti » linguistique et sur la diversité des représentations linguistiques nous ont encouragées à adopter une épistémologie située basée sur la pluralité des points de vue.

\subsection{Folks analyses et quête du juste terme}

Afin de trouver une autodésignation répondant à leur situation de parent endeuillé, les agents énonciatifs effectuent des démarches ressortant des folk analyses dont je rappelle brièvement la typologie tripartite envisagée par Marie-Anne Paveau (2000) : descriptions, prescriptions, interventions. Dans un premier temps, ils effectuent une enquête lexicographique (pratique descriptive) :

Ex. 25 


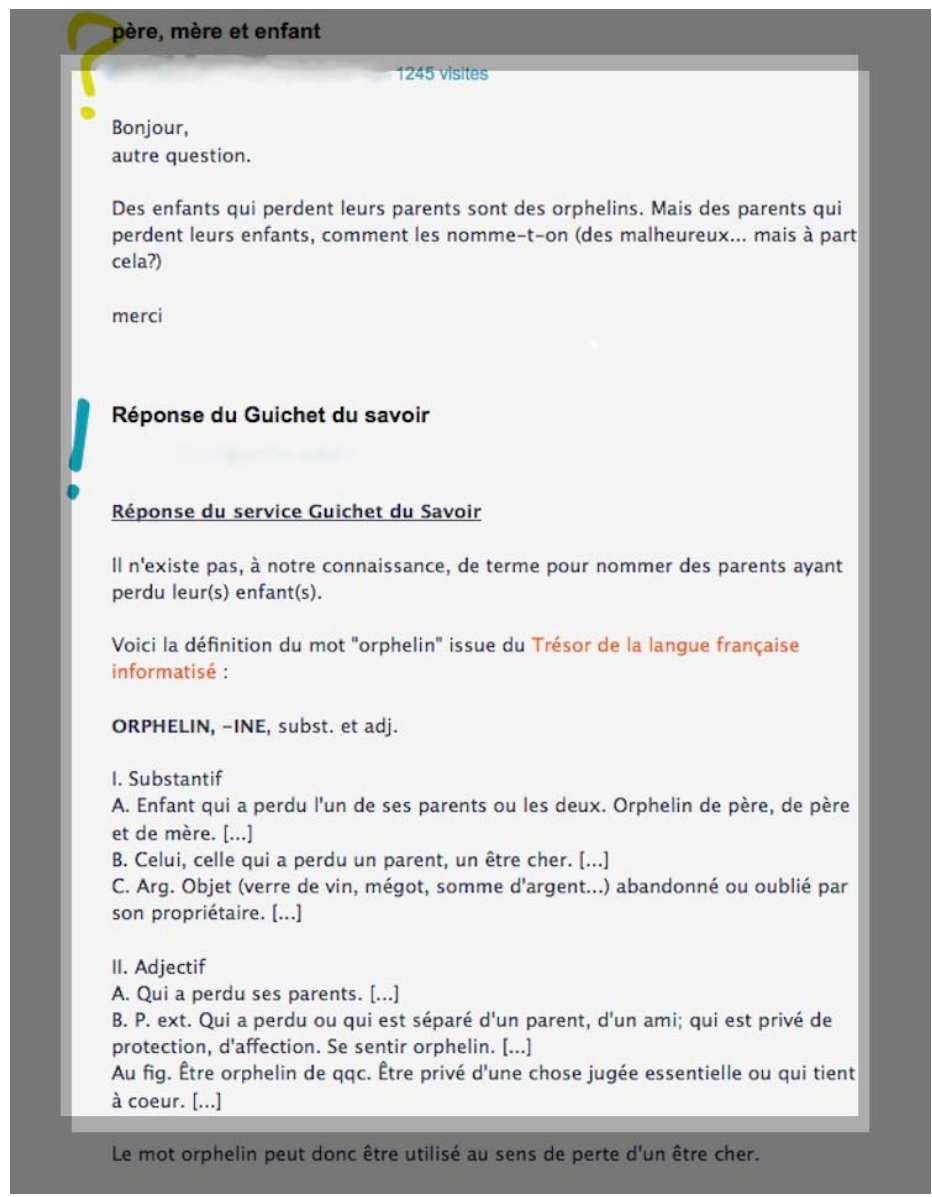

(forum du Guichet du savoir, Orphelin, $1^{\mathrm{er}}$ août 2004, http://www.guichetdusavoir.org/viewtopic.php?t=9907, consulté le 5 janvier 2020)

Dans la discussion ci-dessus (exemple 25), l'agent énonciatif s'est adressé à un organe typique de folk linguistique, le Guichet du savoir. À la question de l'internaute (des parents qui perdent leurs enfants, comment les nomme-t-on), le Guichet du savoir fournit la définition du TLF concernant orphelin. Autrement dit, il s'en réfère aux outils lexicographiques existants. La référence au dictionnaire est typique des folk analyses.

Les parents endeuillés expriment par ces pratiques de linguistique populaire leur vision constructiviste du langage et la nécessité que la langue s'ajuste au monde, par la prise en considération de la mort d'enfant. Il reste qu'elles·ils n'ont pas les moyens académiques d'aller au bout de leur démarche et que seule une politique linguistique accompagnée par des linguistes peut avoir une influence sur ce phénomène de société et aider à lever le tabou autour de la mort de l'enfant. L'objectif ambitieux ici est de faire en sorte que celles et ceux qui n'ont jamais été touchés par une perte similaire, et donc manquent de connaissances expérientielles (Borkman 1976), prennent conscience du phénomène et de ces incidences interactionnelles.

\section{4 Élaborer une «politique linguistique » de nomination d'un point de vue situé : quelques pistes}


Comme nous l'avons précisé, la politique lexicale en France en matière de néologie est attentiste (voir l'exemple 13). Les créations lexicales des parents endeuillés ne sont pas suffisamment diffusées pour qu'elles soient retenues par les instances lexicographiques. En outre, elles ne font pas consensus, comme le montrent les métadiscours des endeuillé·e's et des non endeuillé·e·s. Pourtant, seul un terme adoubé de toutes et tous pourrait démarginaliser les personnes touchées par le deuil d'enfant. C'est l'objet du programme de recherche que nous avons initié, et qui nécessite une méthodologie participative et située.

\subsection{D'un phénomène empirique, le deuil, à une éthique de la nomination}

Les parents endeuillés sont face à des désignations médico-juridico-administratives inappropriées ou dévalorisantes et sont confrontés simultanément à une absence de nomination. Un regard jurilinguistique souligne la difficulté de désigner un enfant décédé précocement par des catégories juridiques telle que «pièces anatomiques d'origine humaine » (circulaire ministérielle du 19 juin 2009 relative à l'enregistrement à l'état civil des enfants décédés avant la déclaration de naissance, qui s'appuie sur les articles R.13359 à R-1335-11 du code de la santé publique et sur les articles 12 et 13 de l'arrêté du 24 novembre 2003). Ces expressions juridiques qui ne catégorisent pas l'enfant décédé dans la classe des êtres humains sont remises en question dans les analyses métalinguistiques des endeuillés. Porteur de différentes valeurs, le terme embryon suscite aussi des désaccords. Lexicalement, il correspond à un stade de développement du corps humain et s'applique jusqu'à douze semaines de gestation. Mais cette appellation aux frontières juridiquement indéterminées désigne aussi bien un être humain que des tissus humains (McGuinness 2012). Or les parents dolents considèrent avoir perdu un bébé et non des tissus humains ou des pièces anatomiques. Ces polémiques mettent en lumière les difficultés à définir la personne humaine. C'est particulièrement sensible dans les travaux parlementaires sur la bioéthique. De leur côté, les Cours d'Appel ont à plusieurs reprises contesté la position de la Cour de Cassation lorsqu'elle retenait la définition juridique d'une personne humaine en tant que « personne vivante »; la Cour Européenne des Droits de l'Homme souligne aussi «l'absence de définition scientifique et juridique des débuts de la vie » et laisse à la libre appréciation des pays membres le soin de déterminer ce point de départ (Roux-Demarre 2012, en ligne). Même l'expression mort-né ne réfère pas à une catégorie homogène (Gourdon et Rollet 2019).

Le problème de nomination de l'enfant décédé est au cœur de l'histoire du droit. Les textes juridiques français recourent notamment au qualificatif innommé pour désigner l'enfant non né. La première occurrence se trouve dans l'arrêt de la cour de Cassation du 7 août 1874 :

\section{Ex. 26}

[...] l'être qui vient au monde avant ce terme [de 180 jours], privé non seulement de la vie, mais des conditions organiques indispensables à l'existence, ne constitue qu'un produit innomé et non un « enfant », dans le sens que le législateur a attaché à cette expression [...].

(Arrêt de la Cour de Cassation, 7 août 1874)

Ce terme, dont l'inadéquation est souvent relevée dans les discours des endeuillés, pointe une spécificité de l'enfant décédé précocement : il n'est pas nommé. La loi ne permet pas qu'il porte un nom de famille et ne le considère pas comme une personne, délégitimant ainsi certaines grossesses. 
Dans la perspective performative de la nomination, nommer fait exister (Austin 1962). La singularisation de l'être social se fait par le nom propre au travers d'actes de langage performatifs oraux transcrits dans les déclarations de naissance et de décès (Austin 1962, Fraenkel 2006, Fracchiolla 2017) et la place dans la famille (inscrite dans le livret de famille). L'être non advenu à la vie, à jamais infans (du latin infantis « qui ne parle pas »), ne peut être confirmé que par la parole d'autrui. Si l'on reprend les catégories de Boltanski (2004) lorsqu'il traite de l'avortement dans sa théorie de l'engendrement et que l'on se permet ici d'appliquer au décès périnatal, les êtres peuvent être engendrés par la chair («fœetus tumoral », embryon accidentel non désiré) ou par la parole des parents («fœtus projet», être «confirmé »). Dans le cas qui nous préoccupe ici, la mort périnatale, les êtres ont été engendrés par la chair et par la parole. En effet, ils peuvent avoir été (pré)nommés à différents moments (avant la conception, pendant la grossesse, après le décès). Cependant, il y a conflit entre le point de vue des personnes qui engendrent (et qui peuvent confirmer comme être singulier l'être non advenu à la vie) et de celui des observateurs extérieurs (pour qui cette assimilation des êtres par la chair à des êtres par la parole ne va pas de soi).

Cette recherche s'inscrit dans ce que nous appelons une "éthique de la nomination » (Ruchon 2018), et qui s'intègre dans l'éthique du souvenir définie par Margalit ([2002] 2006). L'absence d'un nom pour désigner le parent endeuillé soulève le problème moral de la non reconnaissance de l'enfant décédé et de la douleur des parents (Memmi 2011 : 57). L'éthique de la nomination s'appuie sur le concept de sollicitude, employé par Ricœur à propos de l'éthique médicale (Canto-Sperber [1996] 2004 : 695) et sur celui de souci d'autrui (Margalit ([2002] 2006). Le culte des morts est un devoir éthique que s'imposent de nombreuses sociétés mais dans les sociétés modernes, c'est aux institutions de prendre en charge ce travail mnémonique ([2002] 2006) : 24 et 65). L'existence d'un terme permettant aux parents endeuillés de se définir comme tels dans l'interaction permet pragmatiquement aux parents des échanges discursifs avec leurs proches, contraints d'entériner cette existence. Cependant l'acte de nommer ne peut s'exercer librement, il est soumis à des contraintes d'ordre socio-éthiques, ainsi qu'à des contraintes institutionnelles et politiques. Les personnes détenant une certaine forme de pouvoir - personnes de savoir - médecins - institutions - pouvoirs publics - ne sont pas toujours prédisposés à reconnaître ces tentatives désordonnées de nommer et de rendre justice. La lacune lexicale relevée par les parents endeuillés ne peut être comblée que par une co-construction de savoirs critiques.

Comme le défend la théorie de l'hypostatisation (Schmid 2008), l'existence d'un terme permet aux utilisatrices'teurs de la langue de s'approprier des concepts, de créer un lien référentiel avec une entité correspondante. Cela est encore plus nécessaire lorsque l'enfant décédé n'a pas ou peu vécu. Dans le contexte de la mort périnatale, un (nouveau) terme pour désigner le bébé ou ses parents affirme leur réalité, ce qui peut être extrêmement utile dans le processus de deuil ainsi que pour lutter contre la tabouisation de la mort d'enfant. La reconnaissance du statut de parent passe en effet par la reconnaissance de l'existence de 1 'enfant décédé, et donc par sa nomination.

\subsection{Une méthodologie participative}

Nous souhaitons initier une méthodologie qui s'inscrive dans cette éthique de la nomination en nous appuyant sur les épistémologies du folk par une approche relevant à la fois de l'analyse du discours et de l'ethnographie. L'approche ethnographique nous permettra de travailler la question de la nomination de manière située et participative, en intégrant les concerné e-s à la fabrique des savoirs, mais aussi dans cette réciprocité méthodologique qui permettra de nous rendre visibles en tant que chercheuses et analystes. 
Nous parlons en tant que femmes, et femmes ayant perdu des enfants. Nous partageons avec les agents énonciatifs un privilège épistémique (Harding 2004). Mais nous ne considérons pas que cette position nous ouvre un accès privilégié et exclusif au savoir. La mobilisation de l'expérience et la médiation de notre subjectivité ne constituent que l'une des voies d'accès au savoir. Notre souhait épistémologique est d'œuvrer avec les concerné·e's et d'aller vers une " objectivité forte » (Harding 1991, 1995) par la pluralité de points de vue situés, y compris celui d'hommes (en suscitant notamment la prise de parole des pères endeuillés, qui s'expriment très peu sur leur deuil), de personnes non endeuillées, et de «profanes » c'est-à-dire de personnes concernées par le sujet mais ne relevant pas de la sphère universitaire ou scientifique. Il s'agit d'aller vers une science démocratique en impliquant les demandes et les savoirs profanes, en faisant « de l'enquête elle-même un dialogue à plusieurs voix » (Kocadost 2017, en ligne). Nous souhaitons donc présenter aux concerné·e·s l'état des lieux des désignations existantes en France mais aussi des désignations existant dans d'autres parties du monde, par exemple : pais orfaos de lhos en brésilien (« parents orphelins d'enfant ») ; el oumo el makloumatou qui désigne la mère en deuil en arabe ; kurmirî, nom/adjectif qui désigne les parents ayant perdu un garçon (sans distinction de père ou de mère) et qui signifie littéralement « garçon mort » (celui dont le garçon est mort), il semble qu'il n'y ait aucun nom pour les parents qui ont perdu une fille. La seconde phase consistera à la mise en place de séances de réflexion lexicographique sur la création de néologismes pour désigner le parent endeuillé et son enfant.

Cette méthodologie requiert un cadre, un lieu de rencontre avec les personnes endeuillées. Nous envisageons une forme de partenariat participatif avec l'association Nos tout-petits ${ }^{4}$ dont la vice-présidente, Maryse Dumoulin, est l'auteur du premier DEA d'éthique sur le sujet du deuil périnatal (Dumoulin 1998). Cette association organise régulièrement des réunions de groupe de soutien autour du deuil périnatal organisées à la maternité de Lille auxquelles nous participerons.

Nous prolongeons cette étude en soumettant un questionnaire aux parents endeuillés en France ainsi qu'au Royaume-Uni. Nous pensons en effet qu'une étude comparative pourra apporter un éclairage supplémentaire et permettre de mesurer des variables spécifiques dans la sélection d'un ensemble de néologismes possibles. Ce questionnaire a déjà été transmis au Royaume-Uni et va être prochainement diffusé en France. Cette démarche permettra de recueillir des commentaires métalinguistiques au sujet de termes désignant le bébé mort-né ou les parents eux-mêmes et d'évaluer le seuil d'acceptabilité des locutrices'teurs consulté $\cdot e \cdot s$ en fonction de différents critères, et notamment l'attachement à l'étymologie (manifesté dans les métadiscours profanes des endeuillé·e·s comme dans les exemples 17,23) et au code linguistique (exemples 14, 15). Le but est d'identifier une sélection d'expressions linguistiques qui répondent le mieux possible aux besoins émotionnels et sociaux des parents endeuillés.

Ce questionnaire fait état des diverses désignations évoquées supra: les lexies latines et grecque (mater orba, mater dolorosa, orphanè), les auto-désignations françaises (mamange/papange/parange, parents orphelins), les hétérodésignations employées par les non concerné·e·s (parents endeuillés), les désignations étrangères (désignations néerlandophones par exemple, comme sterrenkind (enfant étoile), sterrenouder (parent étoile)), les expressions lexicalisées à particule du type [feu $+\mathrm{Npr}]$.

La réflexion lexicologique s'insérera dans une réflexion plurisémiotique en prenant aussi en compte les expressions grapho-iconiques, sur l'exemple des Pays-Bas qui apposent une étoile aux côtés du nom de l'enfant décédé dans certains documents scripturaux (faire-parts) et les représentations iconiques de l'enfant décédé (avatar ange, étoile et autres éléments cosmologiques).

Enfin, cette enquête sera complétée par des entretiens individuels avec les parents endeuillés (organisés au sein de l'association lilloise Nos tout-Petits). 
Le cadre ethnographique nous permettra de produire des savoirs situés et de faire participer les concernéee-s à l'analyse et à la mise en place d'un terme permettant de les désigner, d'un terme pouvant fonctionner à la fois comme autodésignation et comme hétérodésignation. Il ne s'agit pas cependant de vouloir naïvement aplanir notre position de chercheuses dotées d'une science linguistique face à des locutrices·teurs doté·e-s d'une expertise empirique, mais de faire dialoguer ces savoirs et ces points de vue afin d'élargir l'angle de vue. Nous œuvrons en faveur d'une recherche panoramique, qui embrasse différents points de vue, afin d'apporter des résultats concrets à des problèmes considérés comme indicibles ou insolubles.

\section{Conclusion}

Nous espérons par ces travaux préliminaires sur cette épineuse question de nomination initier une méthodologie participative permettant de recueillir des matériaux en terrain sensible et de les travailler en concertation avec les concerné·e·s.

Ce type de démarche pourrait être reproduit sur l'ensemble des désignations posant des problèmes éthiques telles que bébé médicament ou bébé double espoir (désignations des enfants conçus par sélection d'embryon avec une visée thérapeutique dans le but de soigner un frère ou une sœur), ou enfant arc-en-ciel (désignation des enfants nés après un deuil périnatal), etc.

La prise en compte des lacunes lexicales relève d'une démarche constructiviste. Une désignation des parents endeuillés, acceptée et reconnue par les concerné·e·s et les non concerné·e-s (catégorie réversible, sachant que toute personne non concernée peut en tant que parent, grand-parent, tante ou oncle, être un jour concerné par le décès d'un enfant de la famille) permettrait d'agir contre l'invisibilisation des personnes endeuillées, de lutter contre la doxa religieuse et sociale qui recommande l'acceptation des arguments de la fatalité et des bienfaits du temps. Une juste désignation aiderait in fine à changer le regard sur la mort dans un monde occidental et chrétien qui occulte cette évidence.

\section{Références bibliographiques}

Achard-Bayle, G. (2000). Référents évolutifs et point de vue. Linx, $N^{\circ} 43$, 121-144.

- (2008). Les réalités conceptuelles. Identité et/en Fiction. Recherches textuelles, $N^{\circ} 8$.

Achard-Bayle, G. et Paveau, M.-A. (éd.). (2008). Linguistique populaire ?. Pratiques, N¹39-140.

Austin, J. L. (1962). Quand dire, c'est faire. Trad. de l'anglais par G. Lane. Paris : Le Seuil, 1970.

Authier-Revuz, J. (1990). La non-coïncidence interlocutive et ses reflets méta-énonciatifs. Dans Berrendonner A. et Parret H. (éds), L'interaction communicative. Berne : Peter Lang, 173-194.

Bacqué, M.-H. et Biewener, C. (2013). L'empowerment, une pratique émancipatrice. Paris : La découverte.

Beacco, J.-C. (dir.). (2004). Représentations métalinguistiques ordinaires et discours. Langages, $N^{\circ} 154$.

Boltanski, L. (2004). La condition fretale. Une sociologie de l'engendrement et de l'avortement. Paris : Gallimard.

Borkman, T. (1976). Experiential Knowledge: A New Concept for the Analysis of Self-Help Groups. Social Service Review, $N^{\circ} 50 / 3,445-456$.

Breton, M. (2000). Partenariats et travail communautaire. Revue Informations sociales, $N^{\circ} 83, \mathrm{CNAF}$, $38-49$. 
Bruns, A. (2008). Blogs, Wikipedia, Second Life and Beyond: From Production to Produsage. New York: Peter Lang Publishing.

Cazal, Y. (2009a). Nec jam modo mater, enquête sur une dénomination disparue pour désigner "la mère qui a perdu son enfant ", Micrologus, Nature, Scienze e Società medievali, N¹7, 235-253.

- (2009b). Une lacune qui fait parler : quand le discours spontané sur le lexique s'exerce sur la lacune lexicale : "mère-qui-a-perdu-son-enfant". Dans Achard-Bayle G. et Lecolle M. (éds.), Recherches Linguistiques, $N^{\circ} 30,105-121$.

Chanay, H. de et Rémi-Giraud, S. (2002). “ Espèces d'espaces” : approche linguistique et sémiotique de la métaphore. Mots. Les langages du politique, $N^{\circ} 68,75-105$.

Chachou, I. (2012). L'auto-désignation et l'hétéro-désignation comme procédés langagiers de ségrégation urbaine : le cas de la ville algérienne de Mostaganem. Synergies: Algéries, $N^{\circ} 15$, 169-177.

Collinot, A. et Mazière, F. (1997). Un prêt à parler : le dictionnaire. Paris : Presses universitaires de France.

CSTS (2015). Refonder le rapport aux personnes « Merci de ne plus nous appeler usagers. Rapport $\mathrm{du}$ Conseil supérieur du travail social pour la Direction générale de la cohésion sociale, $<$ https: $/ /$ solidaritessante.gouv.fr/IMG/pdf/CAB COM RAPPORT COMPLET Merci non usagers.pdf $>$.

Canto-Sperber, M. (dir.). ([1996] 2004). Dictionnaire d'éthique et de philosophie morale. Paris: PUF.

Despret, V. (2009). Penser comme un rat, Versailles : Quae.

- (2012). «Penser par les effets. Des morts équivoques. Études sur la mort, vol. 2, $N^{\circ} 142$, 31-49.

- (2019). Habiter en oiseau. Arles : Actes sud.

Dorlin, E. (2008). Sexe, genre et sexualités : introduction à la théorie féministe. Paris: Presses Universitaires de France.

Dumoulin, M. (1998). Respect et considération des corps des fretus décédés. Réflexion éthique. Thèse de doctorat d'université de Santé Publique. Lille.

Fraenkel, B. (2006). Actes écrits, actes oraux : la performativité à l'épreuve de l'écriture. Études de communication, $N^{\circ} 19$, <http:// edc.revues.org/369>

Fracchiolla, B. (2017). Performativité des constructions identitaires : Mariage pour tous, nom, adresse et filiation. Le discours et la langue, $N^{\circ} 9-2,133-144$.

Ghliss, Y., Paveau, M.-A. et Ruchon, C. (dir.). (2019). Dynamiques discursives de la vulnérabilité. Signes, Discours et Sociétés, $N^{\circ} 21$.

Gourdon et Rollet (2009). Les morts-nés à Paris au XIXe siècle: enjeux sociaux, juridiques et médicaux d'une catégorie statistique. Population, vol. 64, 687-722.

Harding, S. (1991). Whose Science, Whose Knowledge? Thinking from Women's Lives. Ithaca: Cornell University Press.

- (1995). "Strong Objectivity": A Response to the New Objectivity Question. Synthese, vol. 104, $N^{\circ} 3,331-349$.

- (2004). A Socially Relevant Philosophy of Science? Resources from Standpoint Theory's Controversiality. Hypatia, vol. 19, $N^{\circ} 1,25-47$.

Haraway, D. (1988). Situated Knowledges: The Science Question in Feminism and the Privilege of Partial Perspective. Feminist Studies, vol. 14, N³, 575-599.

- (2007). Manifeste cyborg et autres essais : sciences, fictions, féminismes. Anthologie établie par Laurence Allard, Delphine Gardey et Nathalie Magnan. Paris : Exils. 
Heazell, A. E. P. (2016). Stillbirth - a challenge for the 21 st century. BMC Pregnancy and Childbirth, $N^{\circ} 16,387-388$.

Hoenigswald, H. (1966). A proposal for the study of folk-linguistics. Dans Bright W. (éd.), Sociolinguistics. Mouton : La Haye, 16-26.

Julia, C. (2001). Fixer le sens ? La sémantique spontanée des gloses de spécification du sens. Paris : Presses Sorbonne Nouvelle.

Kocadost, F. Ç. (2017). Le positionnement intersectionnel comme pratique de recherche : faire avec les dynamiques de pouvoir entre femmes. Les cahiers du CEDREF, $N^{\circ} 21$,

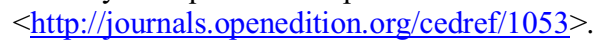

Latour, B. (2012). Enquêtes sur les modes d'existence, une anthropologie des modernes. Paris : La découverte.

Leeman, D. (1995). Pourquoi peut-on dire Max est en colère mais non *Max est en peur ? Hypothèses sur la construction être en $N$. Langue française, $N^{\circ} 105$, 55-69.

Le Bossé, Y. (2004). Vous avez dit "empowerment" ? De "l'habilitation" au "Pouvoir d'Agir" vers une définition plus circonscrite de la notion d'empowerment. Nouvelles Pratiques Sociales, $N^{\circ} 16$ (2).

Margalit, A. ([2002] 2006). L'éthique du souvenir. Paris : Climats.

McGuinness, S. (2012). The construction of the embryo and implications for law. In M. Quigley, S. Chan \& J. Harris (eds), Stem Cells: New frontiers in Science and Ethics, 51-84.

Memmi, D. (2011). La seconde vie des bébés morts. Paris : Éditions EHESS.

Niedzielski, N. et Preston, D. (2003 [2000]). Folk Linguistics. Berlin/New York Boston : Mouton De Gruyter.

Ninacs, W. A. (2002). Types et processus d'empowerment dans les initiatives de développement économique communautaire au Québec. Thèse de doctorat. Université Laval, $<$ http://lacle.coop/docs/ninacs these.pdf $>$, consulté le 20 décembre 2019.

Payet, J.-P. et Laforgue, D. (2008). Qu'est-ce qu'un acteur faible ? Contributions à une sociologie morale et pragmatique de la reconnaissance. Dans Payet J.-P., Giuliani F. et Laforgue D., De l'indignité à la reconnaissance. Enquête sur la voix des acteurs faibles. Rennes: Presses Universitaires de Rennes, 9-25.

Payet, J.-P. (2011). L'enquête sociologique et les acteurs faibles. SociologieS, $<\underline{\text { https://journals.openedition .org/sociologies/3629> }}$, consulté le 20 décembre 2019.

Paveau, M.-A. (2007). Les normes perceptives de la linguistique populaire. Langage et société, $N^{\circ} 119,93-109$.

- (2008). Les non-linguistes font-ils de la linguistique ? Une approche anti-éliminativiste des théories folk. Pratiques, $\mathrm{N}^{\circ} 139-140,93-109$.

Paveau, M.-A. et Perea, F. (2012/2015). Corpus sensibles. Cahiers de praxématique, N559.

Rosier, L. (2004). La circulation des discours à la lumière de "l'effacement énonciatif" : l'exemple du discours puriste sur la langue. Langages, $N^{\circ} 156,65-78$.

- (2008). De la sociolinguistique d'amateur ou Purisme et lutte des Classes. Le français moderne, $N^{\circ} 1,51-65$.

Roux-Demare, F.-X. (2012). L'embryon et le fœtus en droit. Fiche pédagogique Droit civil, Droit pénal, Droit européen. En ligne < http://fxrd.blogspirit.com/media/01/01/1698829562.pdf $>$.

Ruchon, C. (2015). Des vertus antalgiques du discours ? L'expression de la douleur et de l'attachement dans les discours sur la maternité. Thèse de doctorat. Université Sorbonne Paris Cité. 
- (2017). Identité numérique de parents endeuillés. Le pseudonyme comme pratique de deuil. Dans Guilbert T. et Lefort P. (dir.). Discours et (re)constructions identitaires. Villeneuve d'Ascq : Presses Universitaires du Septentrion, 133-148.

- (2018). L'être et le nom : éthique de la nomination dans le cadre du deuil périnatal. Langage \& Société, $N^{\circ} 163,101-119$.

Charolles, M. et Schnedecker, C. (1993, "Coréférence et identité. Le problème des référents évolutifs. Langages, $N^{\circ} 112$, 106-126.

Schmid, H.-J. (2008). New words in the mind: concept-formation and entrenchment of neologisms. Anglia, $N^{\circ} 126(1), 1-36$.

${ }^{1}$ https://www.insee.fr/fr/statistiques/2383444, consulté le 17 janvier 2020

${ }^{2}$ Conformément aux méthodes de citation, la graphie d'origine a été conservée.

${ }^{3}$ Le Figaro, par Alice Develey, 27 avril 2018, “«Parange», un néologisme pour dire le deuil des parents", https://www.lefigaro.fr/langue-francaise/actu-desmots/2018/04/27/37002-20180427ARTFIG00029-parange-un-neologisme-pour-dire-ledeuil-des-parents.php, consulté le 17 janvier 2020.

${ }^{4}$ L'association française Nos Tout-Petits de Lille (http://www.nostoutpetits.fr/, dernier accès : 5 janvier 2020) est constituée de bénévoles et de professionnels de la santé qui offrent leur soutien aux familles qui ont perdu un tout-petit en maternité, pendant la grossesse, ou quelques jours après leur naissance. Cette association collabore aussi avec le Réseau de Santé en Périnatalité de la Région Lilloise OMBREL (https://www.ombrel.fr/, dernier accès : 20 décembre 2019) et a ouvert plusieurs sièges en France : à Nice (Nos ToutPetits de Nice : https://nostoutpetitsdenice.org/, dernier accès : 20 décembre 2019), en Alsace (Nos Tout-Petits d'Alsace : http://nostoutpetitsdalsace.org/, dernier accès : 20 décembre 2019), en Normandie (Nos Tout-Petits de Normandie :

http://www.nostoutpetits.fr/partenariat/nos-tout-petits-de-normandie/, dernier accès : 20 décembre 2019), et en Savoie (Nos Tout-Petits de Savoie : http://nostoutpetitsdesavoie.org/, dernier accès : 20 décembre 2019). Ce partenariat permettra de diffuser les retombées de ce projet (un programme de communication ouverte et respectueuse sur la mort périnatale) audelà des frontières de la Région des Hauts-de-France. 